\title{
The Sun as an X-ray star: Active region evolution, rotational modulation, and implications for stellar X-ray variability
}

\author{
S. Orlando ${ }^{1}$, G. Peres ${ }^{2}$, and F. Reale ${ }^{2}$ \\ 1 INAF - Osservatorio Astronomico di Palermo "G.S. Vaiana”, Piazza del Parlamento 1, 90134 Palermo, Italy \\ e-mail: orlando@oapa.astropa.unipa.it \\ 2 Dip. di Scienze Fisiche \& Astronomiche, Univ. di Palermo, Piazza del Parlamento 1, 90134 Palermo, Italy
}

Received 5 February 2004 / Accepted 14 April 2004

\begin{abstract}
We study the contribution of an active region and its core to the luminosity and the spectrum of the Sun in the X-ray band and to the relevant solar emission measure vs. temperature distribution, $E M(T)$. We also study the relevant changes in the course of four solar rotations, and the solar rotational modulation due to this active region, the only one present at that time. To this end, we have used a large sample of full-disk Yohkoh/SXT observations taken between July and October 1996, covering most of the active region evolution. From the Yohkoh/SXT data we have synthesized the X-ray spectra of the whole solar corona, and the focal plane data as they would be collected with Rosat/PSPC, XMM-Newton/EPIC and Chandra/ACIS. This work is part of a project to study the Sun as an X-ray star, using the solar data as a guide and a template for stellar observations. We found that the active region contributes significantly to the X-ray spectrum of the Sun mainly during the first month of its evolution. The rotational modulation due to the active region causes a significant variability of the average X-ray flux, with only moderate spectral variation, in the pass-bands of Rosat/PSPC, XMM-Newton/EPIC and Chandra/ACIS.

We investigated the characteristics of the X-ray variability due to the rotational modulation, to the solar cycle, and to flares together with the possible implications on stellar X-ray variability. We derived the diagram of X-ray surface flux vs. spectral hardness ratio in the Rosat/PSPC band; we studied the variability due to the solar cycle and to the rotational modulation and we found that both lead to the same correlation between $F_{\mathrm{pspc}}$ and $H R_{\mathrm{pspc}}$ with a very steep slope. The variability due to the evolution of flares again produces a correlation between $F_{\mathrm{pspc}}$ and $H R_{\mathrm{pspc}}$ but with a much flatter slope than in the other two cases. Analogous results have been found when analyzing data in the format of XMM-Newton/EPIC and Chandra/ACIS.
\end{abstract}

Key words. Sun: activity - Sun: corona - stars: activity - stars: coronae

\section{Introduction}

Since the earliest studies of stellar coronae it has been widely agreed that the Sun is a template and a guide to interpret X-ray observations of late-type stars - the so-called "solar-stellar connection".

A template for stellar coronal observations is particularly valuable now, in the light of the high quality X-ray spectra of the new world class instruments, in particular those on board XMM-Newton and Chandra. We have developed a method to translate the spatially resolved solar data collected with the Soft X-ray Telescope (SXT; Tsuneta et al. 1991) on board Yohkoh (Ogawara et al. 1991) into X-ray observations of a star twin to the Sun made with non-solar instruments (Orlando et al. 2000a; Peres et al. 2000; hereafter Papers I and II, respectively). The resulting "stellar" observations of the Sun can be analyzed with the same tools of stellar coronal physics. Yohkoh/SXT data allow us to resolve the regions from which coronal emission originates, to select specific structures, or classes of structures, and to evaluate their contribution to coronal emission.
We have initially applied our method to characterize the Sun at different moments of the activity cycle and during a large flare (Paper II). Then the method has been applied to solar flares (Reale et al. 2001, hereafter Paper III) and to other classes of coronal structures, namely the background quiet corona, active regions and cores of active regions (Orlando et al. 2001, hereafter Paper IV).

In this paper we study the long term evolution of a solar active region from its emergence to the decay phase, distinguishing the contribution of its core from that of the surrounding intermediate brightness region, and of both from the background corona, and analyzing how their relative weights evolve. Detailed studies investigating the properties which characterize the evolutionary behavior of a solar active region are present in the literature (e.g. Golub et al. 1982); at variance with those studies, our main aim here is to investigate the implications of the active region evolution on stellar X-ray variability.

We use Yohkoh/SXT data taken between July and October 1996, when the corona had only one active 
region. Several instruments (including those on board Yohkoh) monitored the evolution of that active region, and the data have been analyzed in detail in several studies (Sterling et al. 1997; Hudson et al. 1998; Harvey \& Hudson 1998; van Driel-Gesztelyi 1998; Oláh et al. 1999; van Driel-Gesztelyi et al. 1999; Orlando et al. 2000b,c; van Driel-Gesztelyi et al. 2003; Dèmoulin et al. 2003). In particular, Oláh et al. (1999) analyzed radio and X-ray data of this active region to model the Sun as a star but with a different aims than in the present paper: they provided diagnostics of photometric star-spot models through modeling active areas on the Sun using tools originally devised for star-spot modeling. This region, therefore, represents an important and useful template for coronal physics. Since many whole disk instruments detected primarily this region (see Sect. 2), in this specific work we are able to include information obtained from the Bragg Crystal Spectrometer (BCS; Culhane et al. 1991; Lang et al. 1992) on board Yohkoh.

We study the evolution of the emission measure vs. temperature distribution, $\operatorname{EM}(T)$, of the X-ray spectrum of the active region, and of its contribution to the whole solar $E M(T)$ and $\mathrm{X}$-ray spectrum. We also investigate the rotational modulation induced by the active region on the solar X-ray luminosity, on the relevant $\operatorname{EM}(T)$ and on the stellar-like spectra. Finally, we compare the characteristics of the stellar X-ray variability due to different coronal phenomena (i.e. rotational modulation, solar cycle, flares) and discuss their implications for stellar X-ray observations.

The paper is structured as follows: Sect. 2 describes the sample of data selected and the method to study the Sun as an X-ray star; Sect. 3 describes the evolution of the $E M(T)$ of the active region, and of its core, during the emergence and the following decay, and analyzes the variability induced by both the active region evolution and the rotational modulation in the stellar-like spectrum of the Sun; in Sects. 4 and 5 we discuss the implications for stellar X-ray variability and draw our conclusions.

\section{The data and their analysis}

The selected sample of Yohkoh observations was collected between July and October 1996, during the minimum of solar cycle 22 . At that time, the X-ray solar activity was dominated by only one active region, designated AR 7978, 7981, 7986 during successive solar rotations (we will refer to it as AR 7978). Since the emission from other coronal structures is negligible for most of the evolution of this active region, whole-disk instruments (i.e. without spatial resolution) such as Yohkoh/BCS responded primarily to this source of activity, and BCS data could, therefore, be included in our analysis.

Transient events (or even periods during the decay phase of flares) were carefully screened out by singling them out in the continuous light curves provided by the X-ray photometers on board the GOES-9 spacecraft $^{1}$ (Geosynchronous Operational Environmental Satellite). SXT and BCS data simultaneous to

\footnotetext{
${ }^{1}$ The GOES spacecrafts have two different X-ray sensors (XRS) capable of providing whole-Sun X-ray fluxes in the 0.5 -to- $4 \AA$ and 1-to-8 Å spectral bands respectively.
}

flares above GOES class B, or to evident GOES flux enhancements of the active region flux, have been discarded.

\subsection{The Yohkoh/SXT data}

The Soft X-ray Telescope (Tsuneta et al. 1991) is a grazing incidence telescope of $1.54 \mathrm{~m}$ focal length which forms X-ray images in the $3-70 \AA$ range on a $1024 \times 1024$ virtual phase CCD detector. A selection of thin metallic filters located near the focal plane provides the capability to separate different $\mathrm{X}$-ray bands for plasma temperature diagnostics.

We selected a sample of 78 full-disk observing sequences collected with the SXT, covering most of the evolution of AR 7978 from July 5, 1996 to the end of October 1996. Our work has a two-fold aim: i) to study the effect of the evolution of the active region; and ii) to study the rotational modulation induced by the active region. For the former investigation, we have taken the data at the emergence of AR 7978 (July 5-13, 1996) and at its next five Central Meridian Passages (CMPs); for the other study, we have used data sampled with a regular cadence of four days. Figure 1 shows the light curve of the whole solar corona in the SXT thin aluminum (Al $1265 \AA$, referred to as Al.1) filter band obtained from the synoptic observations (Morrison 1994). The variability due to the rotational modulation is evident as quasiperiodic changes in the light curve with period of $\approx 27$ days. Though the active region was visible on the disk until the end of December 1996, our selected data stop at the end of October 1996 because after that new active regions emerged on the disk.

The 78 observing sequences are all outside flares and include images with different exposure times $(\sim 78,2600$, and $10700 \mathrm{~ms}$ ) in order to cover the large dynamic range of the corona X-ray brightness. The images were taken through the Al.1 filter and the $\mathrm{Al} / \mathrm{Mg}$ sandwich filter (comprising Al $2930 \AA$, Mg $2070 \AA$, Mn $562 \AA$, and C $190 \AA$, referred to as $\mathrm{AlMg}$ ); this allows us to derive maps of temperature (between $\sim 3 \times 10^{5} \mathrm{~K}$ and $\sim 10^{7} \mathrm{~K}$ ) and of emission measure (Tsuneta et al. 1991; Papers I and II).

\subsection{The Yohkoh/BCS data}

The Bragg Crystal Spectrometer (Culhane et al. 1991) consists of two bent crystal spectrometers, BCS-A and BCS-B, that observe the H-like line complex of $\mathrm{Fe}$ XXVI (1.7636-1.8044 $\AA$ ), and the He-like complexes of Fe XXV

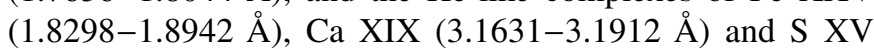
(5.0160-5.1143 $\AA$ ). Each spectrometer consists of a double detector placed behind a pair of germanium crystals that diffract the incoming X-rays into the detectors. BCS has no spatial resolution.

We used helium-like sulfur (S XV) spectra collected quasisimultaneously (within two minutes) with the SXT data to study the hotter plasma of the active region during its emergence (July 5-13, 1996). Most of the X-ray flux during the period of our data came from AR 7978, and we can assume that the above lines came almost exclusively from the active region. 


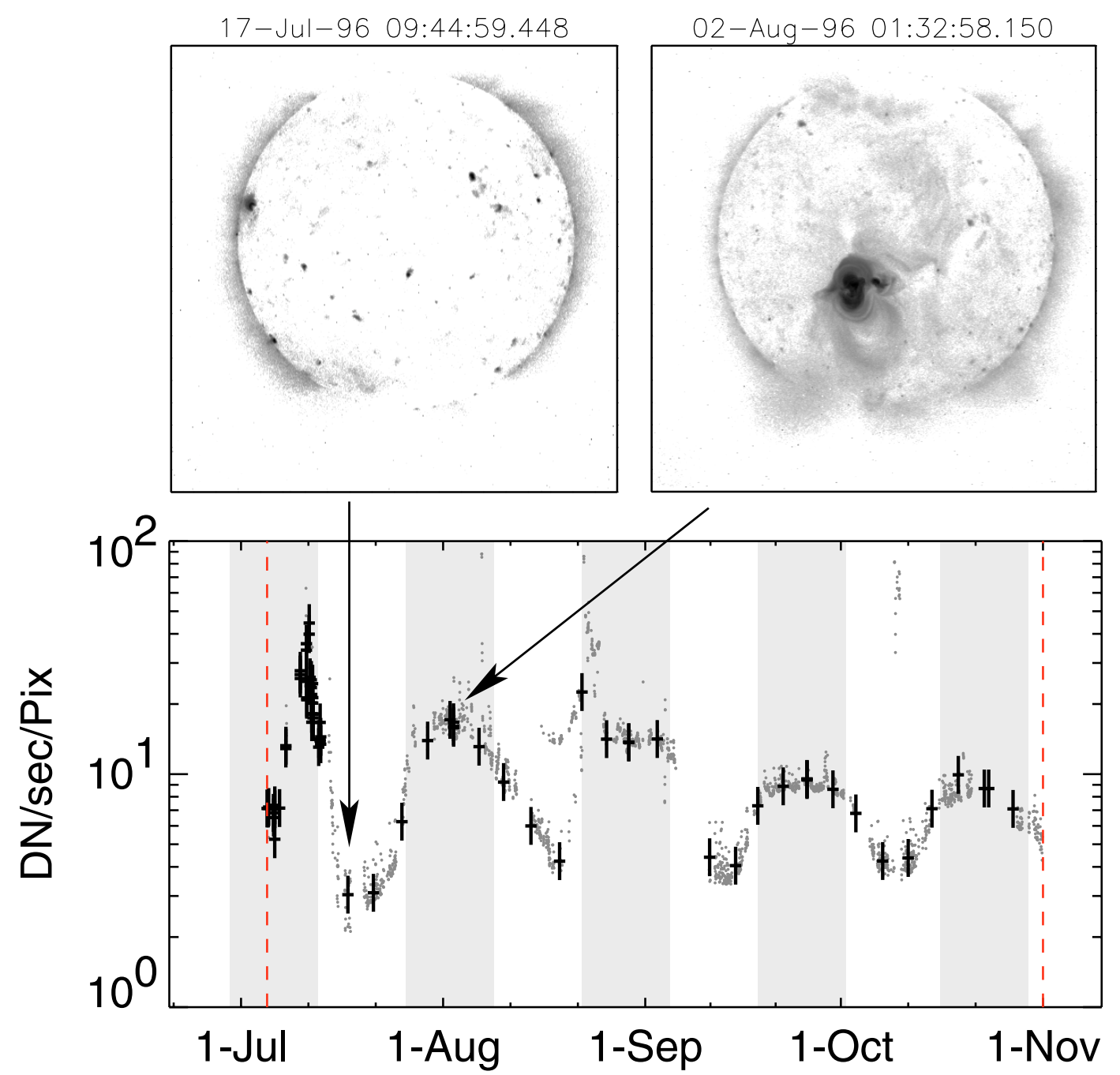

Fig. 1. Upper panels: two examples of SXT full-disk images in the thin aluminum (Al.1) filter band, when the active region was occulted (left) and when the active region was visible on the disk (right). Bottom panel: SXT light curve through the same filter taken from synoptic observations (Morrison 1994). The crosses mark the datasets we have selected for our analysis. The dashed lines bound the total period analyzed (July 5-October 31, 1996) and the gray stripes mark the times when the active region was visible on disk.

The S XV channel is the only BCS channel routinely detecting flux from non-flaring active region plasma.

\subsection{Analysis of the Yohkoh/SXT data}

The SXT data were first processed according to the standard Yohkoh analysis: we performed standard corrections (dark current subtraction, pin-hole visible stray light subtraction), aligned the images, removed the spikes, and applied the vignetting correction. Then with our method (see Papers I and II for more details) we derived the $E M(T)$ distributions of the whole solar corona (or of parts of it) and the related stellar-like spectra. The method consists of four main steps:

1. from two nearly simultaneous (within 3 min) X-ray images collected with SXT, we derive temperature and emission measure for each pixel in the field of view, i.e. a map of coronal temperature and a matching map of coronal plasma emission measure. Saturated and underexposed pixels are taken care of, by combining long and short exposures taken less than 10 min apart;

2. from the temperature and emission measure maps, we derive a distribution of emission measure vs. temperature, $\operatorname{EM}(T)$, of the whole solar corona or of its parts: we divide the nominal temperature range $(5.5 \leq \log T(\mathrm{~K}) \leq 8)$ to which SXT is sensitive into 29 bins, all equal on a logarithmic scale and appropriate to the spectral discriminating power of SXT; the emission measures of all the pixels belonging to the same temperature bin are then summed;

3. from the $\operatorname{EM}(T)$ distribution, we synthesize the $\mathrm{X}$-ray spectrum of the solar corona, using the MEKAL spectral synthesis code (Mewe et al. 1985; Kaastra 1992, and subsequent upgrades);

4. folding the X-ray spectra through the instrumental response of the non-solar instruments of interest, we obtain the relevant stellar-like focal plane spectra.

The final products of our method are new solar X-ray data in a format virtually identical to that of stellar observations 
collected with non-solar X-ray instruments like those on board Rosat, XMM-Newton, and Chandra. To such data, we can apply the standard methods of analysis used for stellar observations and we can compare the results directly with those of stellar X-ray studies.

In this work, all the $\operatorname{EM}(T)$ distributions derived from sets of less than 100 SXT pixels were also excluded from our analysis because of statistical uncertainties, except for the core, for which we accepted in the analysis $E M(T)$ distributions derived from more than 3 SXT pixels, instead of 100, since the core of a single active region can be very small on the image. To analyze the simulated focal plane Rosat/PSPC, XMM-Newton/EPIC, and Chandra/ACIS spectra, we used the spectral fitting package XSPEC V11.2 (Arnaud 1996), routinely used in stellar physics, and applied a multi-temperature fit to each spectrum. We also applied corrections and methods typical of stellar X-ray studies. In the analysis of the Rosat/PSPC spectra, the two lowestenergy channels were excluded because they are typically affected by systematic errors in real observations. The channels with too low signal (less than 3 photons) were also excluded for an appropriate evaluation of $\chi^{2}$ (see Paper II). In the analysis of XMM-Newton/EPIC and Chandra/ACIS spectra, the channels below $0.3 \mathrm{keV}$ and $0.5 \mathrm{keV}$, respectively, were omitted and the energy channels were grouped together so as to have at least 20 photon counts per channel.

Note that also the intermediate results are important by themselves for solar and stellar studies: for example, the $E M(T)$ distributions show the contribution of the coronal plasma at different temperatures to the X-ray emission and they can be compared with those derived from the analysis of stellar observations (see, for example, Drake et al. 2000).

\subsection{Analysis of the Yohkoh/BCS data}

The BCS data were pre-processed according to the standard Yohkoh analysis: we applied the dead-time corrections, the crystal curvature corrections, and the flux calibrations. Then, for S XV spectra, we integrated the data in time bins of $100 \mathrm{~s}$ to increase the signal to noise ratio.

The prominent $\mathrm{S} X \mathrm{~V}$ lines of interest are the resonance line at $5.04 \AA$, the line complex near $5.05 \AA$, and the line complex near $5.10 \AA$ consisting of two dielectronic lines and a forbidden line. We fitted the S XV spectra using the standard Yohkoh programs (Fludra et al. 1989). The data were fitted with single temperature model spectra, which provide the effective electron temperature, $T_{\mathrm{S} \mathrm{XV}}$, and the emission measure, $E M_{\mathrm{S} \mathrm{XV}}$, of the active region in the $S \mathrm{XV}$ band. Note that the temperature derived from the intensity ratio of the blended features (forbidden line and dielectronic satellites) to the resonance line is weighted by the plasma emission measure and by the S XV line emissivity (McCann \& Keenan 1988). As a consequence the contribution from the very hot plasma in the active region has a large weight in the average temperature, $T_{\mathrm{S} X V}$.

Sterling et al. (1997) analyzed the BCS data collected between July 14 and 25, 1996, namely when AR 7978 rotated beyond the solar limb, and they showed that the background spectrum measured on July 20, 1996 (just when AR 7978 was totally occulted and no other active regions were present on the solar disk) has no clear spectral lines and that its flux level is well below that of the active region: any line observed, therefore, originated from the active-region corona.

\section{Results}

\subsection{The $E M(T)$ distribution during the evolution of the active region}

We adopted the same criterion as in Paper IV to identify and to select the active region and its core; the criterion is based on the surface brightness $I$ in each pixel. In particular, in Paper IV, we derived the histogram of intensity values in the pixels of all the composite images in the filter band Al.1 of the sample analyzed; the intensity range was divided into 50 bins of constant $\delta \log I \sim 0.1$. The histogram is characterized by a rise to a maximum value, followed by a decrease according to a power law and, then, by a much steeper fall. With the objective of selecting solar regions using their intrinsic properties, we have chosen, as thresholds, the maximum, at $I=40 \mathrm{DN} / \mathrm{s} / \mathrm{pix}$, and the "knee" in the descent at $I=6000 \mathrm{DN} / \mathrm{s} / \mathrm{pix}$. These are the parameters that discriminate between different surface brightness values: all the pixels with $I \leq 40 \mathrm{DN} / \mathrm{s} /$ pix belong to the background corona, those with $I>6000 \mathrm{DN} / \mathrm{s} /$ pix to the core of the active region, and those with $40 \mathrm{DN} / \mathrm{s} / \mathrm{pix}<I \leq$ $6000 \mathrm{DN} / \mathrm{s} /$ pix to the intermediate brightness portion of the active region (hereafter AR).

Our data sample allowed us to derive more than one $E M(T)$ distribution per day and, therefore, to obtain an average $\operatorname{EM}(T)$ distribution for each day. Figure 2 shows such an average $E M(T)$ distributions of AR 7978 and of its core obtained at emergence and after five consecutive central meridian passages (CMPs). The $E M(T)$ of the AR is initially contained within a narrow temperature range centered at $\sim 2 \times 10^{6} \mathrm{~K}$ and has a steep rise and fall vs. temperature; afterwards, the $E M(T)$ grows, as well as the amount of hot plasma $\left(>3 \times 10^{6} \mathrm{~K}\right)$; its shape remains more or less unchanged for three CMPs (July 10-August 29, 1996); then the AR begins to decay, and the $E M(T)$ distribution gradually decreases and its high temperature descending slope becomes steeper. The core is present, and yields an $\operatorname{EM}(T)$, only during the first three solar rotations (July 7-August 29, 1996): its $E M(T)$ grows rapidly in few days (July 7-10, 1996), centered at a relatively high temperature $\left(\sim 4 \times 10^{6} \mathrm{~K}\right)$; then it decays in two months, gradually cooling and getting narrower.

As shown in Paper IV, useful figures to characterize the $E M(T)$ distribution and its global evolution are the emissionmeasure-weighted temperature and the total emission measure, respectively,

$$
\begin{aligned}
& \langle T\rangle=\frac{\sum_{i} T_{i} E M_{i}}{\sum_{i} E M_{i}} \quad i=1, \ldots ., N_{\text {bin }} \\
& E M_{\text {sum }}=\sum_{i} E M_{i}
\end{aligned}
$$

where $T_{i}$ and $E M_{i}$ are the central temperature and the emission measure value in the $i$ th bin of the $E M(T)$ distribution, respectively, and $N_{\text {bin }}$ is the number of bins. 

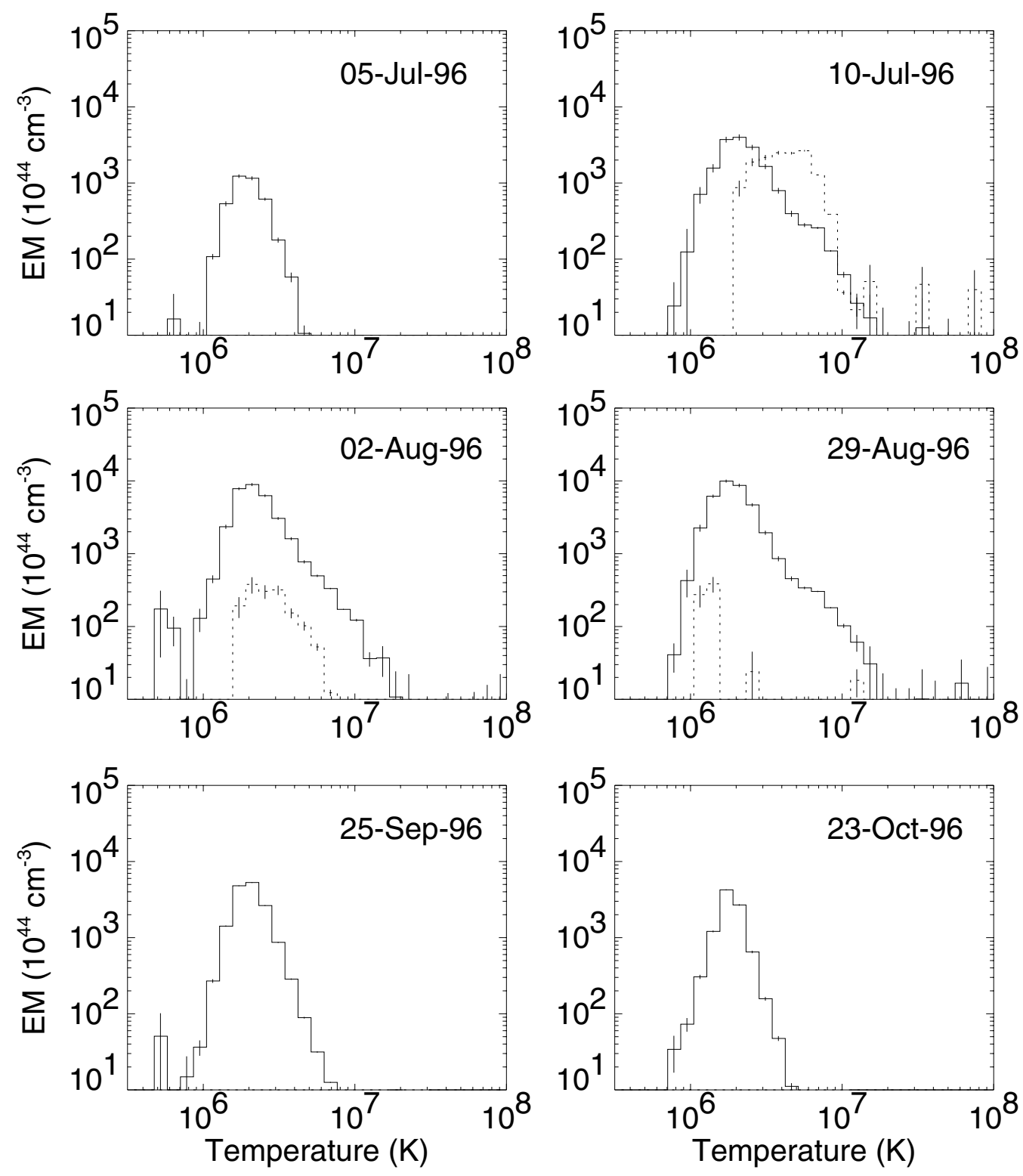

Fig. 2. Left to right and top to bottom, the evolving $E M(T)$ distributions of the intermediate brightness portion of AR 7978 (solid line) and of its core (dotted line) at emergence (upper left panel) and the successive five central meridian passages of the active region.

Figure 3 shows the long term evolution of $\langle T\rangle$ and $E M_{\text {sum }}$ at emergence and at all the CMPs of the active region and of the core. Figure 4 shows an enlargement with higher detail and time resolution of the emergence of AR 7978 in the time range July 5-13, 1996; it will be discussed later.

The temperature $\langle T\rangle$ of the $\mathrm{AR}$ (panel a) increases during its emergence, reaching a maximum $\langle T\rangle \sim 2.5 \times 10^{6} \mathrm{~K}$ around July 10, 1996, and then it slowly decreases following the decay of the active region. The total emission measure has a similar trend: it first increases by a factor $\sim 7$ from July 5 to August 29, 1996, when the $E M_{\text {sum }} \sim 4 \times 10^{48} \mathrm{~cm}^{-3}$ is maximum, and then decreases by one order of magnitude in the course of two rotations. Note that the $E M_{\text {sum }}$ reached its maximum two months later than $\langle T\rangle$, a behavior shared with much more dynamic events, such as flares (e.g. Svestka 1976), but occurring on very different time scales and, probably, involving different physical effects.
Figure 3 also shows the average values of $\langle T\rangle$ and $E M_{\text {sum }}$ of the whole solar corona in the period analyzed (dashed and dotted lines, respectively). We note that the values of $E M_{\text {sum }}$ of the AR are considerably lower than those of the whole corona so that the contribution of the active region to the $\langle T\rangle$ of the whole corona is small. On the other hand, the larger the number of active regions, the larger their influence on $\langle T\rangle$ of the whole corona. In fact, Paper IV shows that $\langle T\rangle$ of the whole corona is highest $\left(\sim 2 \times 10^{6} \mathrm{~K}\right)$ when the $E M_{\text {sum }}$ of the active regions visible on the disk is comparable to, or larger than, the $E M_{\text {sum }}$ of the background corona; this happens, however, at intermediate and high activity phases of the solar cycle.

The core evolves very rapidly when the active region is at the peak of its activity (July-August 1996): when it is visible, its $\langle T\rangle$ decreases from $\sim 4 \times 10^{6} \mathrm{~K}$ to $\sim 10^{6} \mathrm{~K}$ and its $E M_{\text {sum }}$ from $\sim 10^{48} \mathrm{~cm}^{-3}$ to $\sim 10^{47} \mathrm{~cm}^{-3}$. Figures 2 and 3 shows that the core contribution to the whole corona $\langle T\rangle$ is 

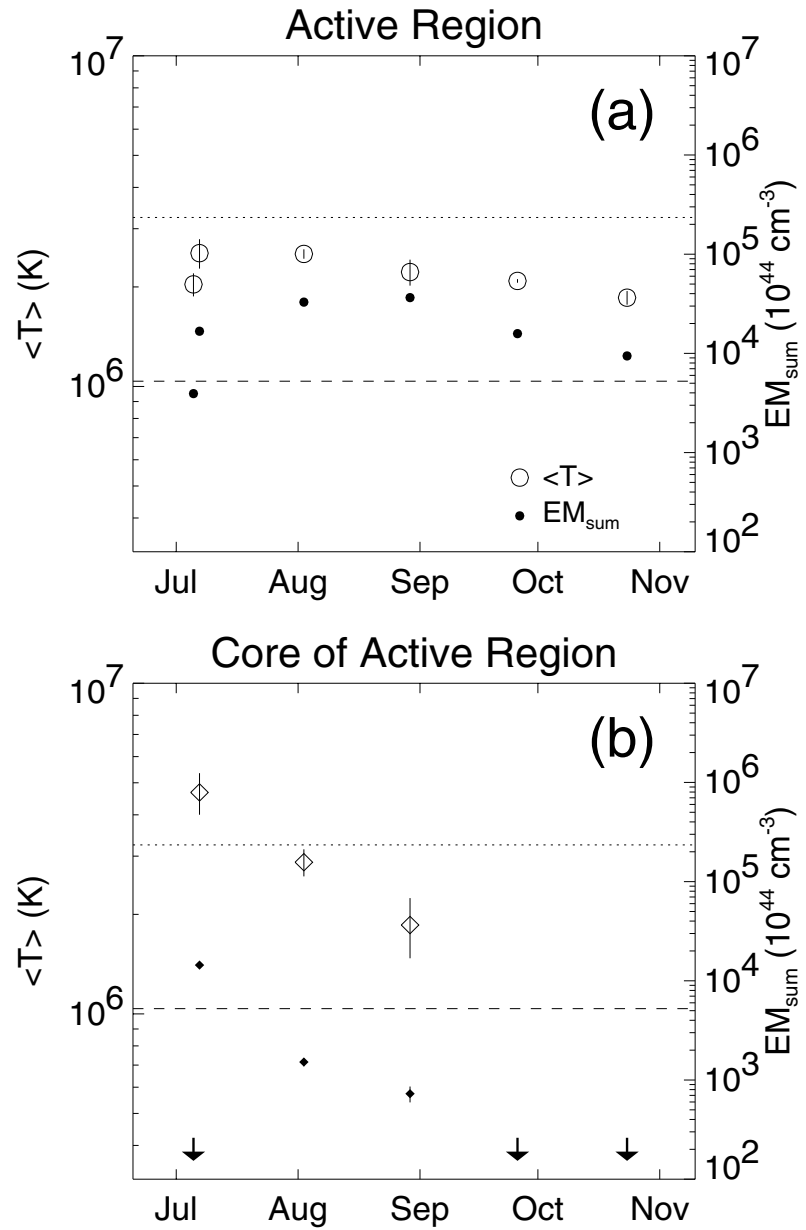

Fig. 3. Evolution of the emission-measure-weighted temperature $\langle T\rangle$ (open symbols) and of the total emission measure $E M_{\text {sum }}$ (filled symbols) at the emergence of the active region and at its consecutive central meridian passages. Panel a), the intermediate brightness portion of the active region (circles); panel $\mathbf{b}$ ), the core of the active region (diamonds). Error bars are shown and may be smaller than symbol size. The dashed and the dotted lines mark $T \sim 1.04 \times 10^{6} \mathrm{~K}$ and $2.3 \times 10^{49} \mathrm{~cm}^{-3}$, respectively, corresponding to the average values of the whole solar corona in the period analyzed. The arrows in panel b) mark observations having too few pixels to determine the $E M(T)$ distribution.

significant just after the emergence of the active region (July 10, 1996).

We note that, although the contribution of the active region and of its core to the $E M_{\text {sum }}$ of the whole corona is small, the active region leads to a well observed rotational modulation of the full-disk X-ray flux in the SXT band (see Fig. 1). This behavior is explained by noting that SXT is very sensitive to the relatively hot plasma of the corona and that its sensitivity is poor for plasma at $T<10^{6} \mathrm{~K}$. The integrated full-disk emission through the SXT Al.1 filter is therefore dominated by the plasma at temperatures around $3 \times 10^{6} \mathrm{~K}$ (see also Tsuneta et al. 1991). Since most of the plasma at that temperature comes from the active region, the SXT light curve shown in Fig. 1 is very sensitive to the rotational modulation.

We study the evolution of the core in more detail in the 42 sequences of full-disk SXT images between

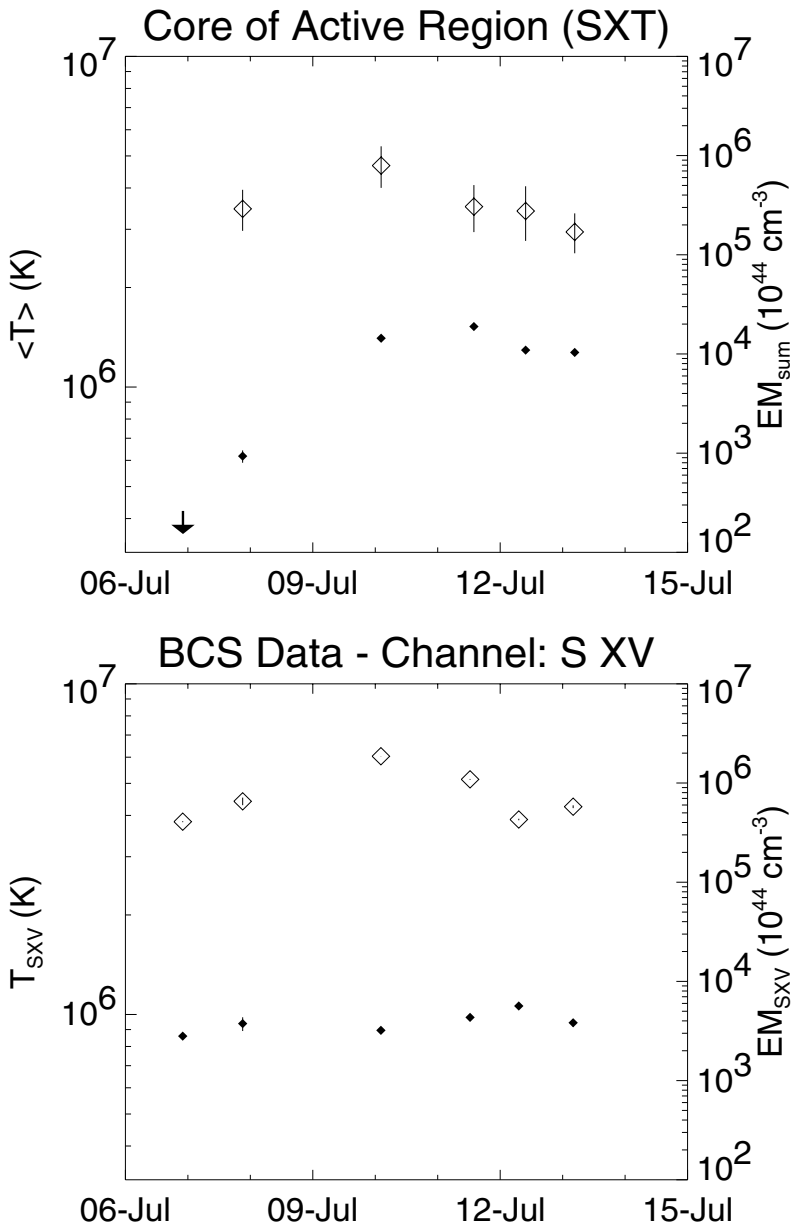

Fig. 4. The evolution of $\langle T\rangle$ and $E M_{\text {sum }}$ of the core (upper panel) away from transient events during the emergence of the active region (July 5-13, 1996). The lower panel shows the temperature, $T_{\mathrm{S} \mathrm{XV}}$, and emission measure, $E M_{\mathrm{S} \mathrm{XV}}$, derived from the data collected with BCS in the S XV channel and averaged over each day. Symbols and labels as in Fig. 3.

July 5 (00:20 UT) and July 13 (21:21 UT), 1996. Figure 4 shows the rapid evolution of $\langle T\rangle$ and $E M_{\text {sum }}$ of the core in this time interval, including the results obtained from BCS data. At the very emergence of the active region (July 5-7, 1996), there are too few core pixels for the analysis. The earliest core $\langle T\rangle$ is already high and increases slightly reaching the maximum on July 10, 1996; then it gradually decreases from $\sim 4.7 \times 10^{6} \mathrm{~K}$ to $\sim 3 \times 10^{6} \mathrm{~K}$ in the period July $10-13,1996$. The corresponding $E M_{\text {sum }}$ starts low, around $10^{47} \mathrm{~cm}^{-3}$, on July 7, 1996; it reaches a maximum, between 1.4 and $1.9 \times 10^{48} \mathrm{~cm}^{-3}$ on July $10-11,1996$, and it decreases slightly to $\sim 10^{48} \mathrm{~cm}^{-3}$ on July $12-13,1996$. The $E M_{\text {sum }}$ is around $2 \times 10^{47} \mathrm{~cm}^{-3}$ when the region appears again on the disk on August 2, 1996, and the corresponding $\langle T\rangle$ is around $3 \times 10^{6} \mathrm{~K}$.

The BCS data simultaneous (within $2 \mathrm{~min}$ ) to the SXT observing sequences allow us to study the hot plasma of the active region (mainly located in the core) and to complement our SXT findings. Figure 4 shows the daily averaged $E M_{\mathrm{S} X V}$ and the corresponding emission-measureweighted $T_{\mathrm{S} X V} . T_{\mathrm{S} \text { XV }}$ first increases from $\sim 4 \times 10^{6} \mathrm{~K}$ to $\sim 6 \times$ $10^{6} \mathrm{~K}$ between July 6 and 10, 1996, reaches a maximum around 
July 10, 1996, and then decreases in the next two days. The BCS data therefore confirm the general trend of the average temperature of the hot plasma component of the active region, first ascending and reaching the maximum on July 10, 1996, and then descending between July 10 and July 13, 1996. The $T_{\mathrm{S} \mathrm{XV}}$ is constantly $\sim 30 \%$ higher than $\langle T\rangle$ of the core, as BCS is more sensitive to hot plasma than SXT. At variance with SXT, $E M_{\mathrm{S} X V}$ does not show any clear evolution and stays within values of 3 and $6 \times 10^{47} \mathrm{~cm}^{-3}$. As a consequence, the ratio $E M_{\mathrm{S} X \mathrm{XV}} / E M_{\text {sum }}^{\text {core }}$ is larger than 1 before July 10,1996 , it is around 0.2 between July 10 and 11,1996 , and it slightly increases to $\sim 0.5$ in the next days. $E M_{\mathrm{S} \mathrm{Xv}} / E M_{\text {sum }}^{\text {core }}>1$ may be explained if, at the emergence of the active region, its core has dimensions comparable to or smaller than the SXT pixel size. Since the analysis of SXT data yields the line-of-sight averaged isothermal temperature and emission measure in each pixel, a significant fraction of the core may be averaged with the rest of the active region and, therefore, is not detected with SXT.

\subsection{The stellar-like spectra and their analysis}

The $\operatorname{EM}(T)$ distributions derived from the SXT data have been used to synthesize the corresponding X-ray spectra in the $0.1-10 \mathrm{keV}$ band, using the MEKAL spectral code. These spectra have been folded through the instrumental response of Rosat/PSPC, XMM-Newton/EPIC, and Chandra/ACIS, yielding the focal plane spectra that these instruments would collect when observing a star identical to the Sun. We assumed that this Sun-like star is at a distance of $1 \mathrm{pc}$ and for each spectrum we adjusted the exposure time in order to collect a total number of photons typical of a good observation and adequate for a spectral analysis; more specifically $\sim 4000$ photons with Rosat/PSPC, and $\sim 10000$ photons with XMM-Newton/EPIC and Chandra/ACIS.

The resulting Rosat/PSPC spectra are well fitted with one or two isothermal components (the reduced $\chi^{2}$ never exceeds 1.1 ): only one component is sufficient in some cases because of their narrow $\operatorname{EM}(T)$ distributions. The multi-isothermal component fitting of the XMM-Newton/EPIC and Chandra/ACIS spectra has shown that at least two components are necessary to fit the spectra and, in some cases, the XMM-Newton/EPIC spectra require a third component.

From the synthesized spectra, we derived $F_{\mathrm{X}}$, the $\mathrm{X}$-ray surface flux in the instruments' spectral band $(0.1-3 \mathrm{keV}$ for Rosat/PSPC; $0.3-10 \mathrm{keV}$ for XMM-Newton/EPIC; and $0.5-10 \mathrm{keV}$ for Chandra/ACIS) and the observed spectral hardness $H R$, important for characterizing the stellar activity and its evolution, both short and long term (see, for example, Schmitt 1997; Micela et al. 1997; and Peres et al. 2004). The spectral hardness in the spectral band of the instrument is defined as

$H R=\frac{H-S}{H+S}$

where $S$ and $H$ are the total counts in the soft and the hard part of the spectrum, respectively. For Rosat/PSPC the soft band is $0.13-0.40 \mathrm{keV}$ and the hard band is $0.55-1.95 \mathrm{keV}$; for XMM-Newton/EPIC, the soft band is $0.3-0.9 \mathrm{keV}$ and the hard band is $0.9-3.5 \mathrm{keV}$; and for Chandra/ACIS, the soft band is $0.5-0.9 \mathrm{keV}$ and the hard band is $0.9-3.5 \mathrm{keV}$. The hardness ratio is a rough but quite effective indicator of the average temperature of the emitting plasma. We defined the X-ray surface flux, $F_{\mathrm{X}}$, dividing the X-ray luminosity in the instrument band (computed from the fitted isothermal components) by the area taken in the SXT images of the corresponding selected region. Note that, in the case of the whole corona, the surface area is that of the whole Sun. Note also that both $H R$ and $F_{\mathrm{X}}$ are different for different instruments because of the different spectral bands.

\subsubsection{Active region evolution}

The evolution of $H R$ and of $F_{\mathrm{X}}$, in the Rosat/PSPC, XMM-Newton/EPIC, and Chandra/ACIS bands has been analyzed for the whole corona, the AR and the core at the emergence of the AR 7978 and at its successive five CMPs. Figure 5 shows representative results obtained for XMM-Newton/EPIC. The results for the other instruments are analogous.

Figure 5 shows that the $H R_{\text {epic }}$ of the whole corona increases from $\sim-1.0$ to $\sim-0.8$ at the emergence of AR 7978; then it slowly decreases to $H R_{\text {epic }} \sim-0.95$ at the end of October 1996. The corresponding flux, $F_{\text {epic }}$ is confined in a narrow range of values between $2 \times 10^{3}$ and $4 \times$ $10^{3} \mathrm{erg} \mathrm{cm}^{-2} \mathrm{~s}^{-1}$ with a moderate maximum on August 2, 1996.

The $H R_{\text {epic }}$ and $F_{\text {epic }}$ of the intermediate brightness portion of AR 7978 indeed follow a similar and even better-defined evolution: $H R_{\text {epic }}$ rapidly increases from -0.92 to -0.83 at the emergence of AR 7978 (July 5-10, 1996), and then slowly decreases to -0.94 at the end of October, 1996; $F_{\text {epic }}$ increases from $8 \times 10^{4}$ to $1.3 \times 10^{5} \mathrm{erg} \mathrm{cm}^{-2} \mathrm{~s}^{-1}$ during the first solar rotation (July 5-August 2, 1996), and then decreases to $3 \times 10^{4} \mathrm{erg} \mathrm{cm}^{-2} \mathrm{~s}^{-1}$ at the end of the decay phase (end of October 1996).

Figure 5 also shows the $H R_{\text {epic }}$ and $F_{\text {epic }}$ values of the core during the emergence of the active region. The $H R_{\text {epic }}$ increases to $\sim-0.65$, in July 10,1996 , when the active region is at the peak of its activity, it decreases to $\sim-0.8$ in the following two days, then it slowly decreases further in the next month. The corresponding $F_{\text {epic }}$ instead is almost constant, steadily $>10^{6} \mathrm{erg} \mathrm{cm}^{-2} \mathrm{~s}^{-1}$ (with a maximum of $3 \times$ $10^{6} \mathrm{erg} \mathrm{cm}^{-2} \mathrm{~s}^{-1}$ ). The active region and its core mainly influence the spectral hardness of the whole solar corona at the emergence of AR 7978, when their hardness ratio is maximum; they mainly influence the flux $F_{\text {epic }}$ one month after the emergence, when their flux is maximum.

During the maximum and intermediate phases of the solar activity, several active regions are present simultaneously in the corona, each of them in a different phase of its evolution: the global $H R_{\text {epic }}$ and $F_{\text {epic }}$ reflect the weighted average of various ARs at different phases of their evolution. The present analysis tells us that the average contribution of the active regions to the whole corona X-ray spectrum is dominated by the young regions with high $F_{\text {epic }}$ : in agreement with Peres et al. (2004), the X-ray spectrum of the Sun during the maximum of 

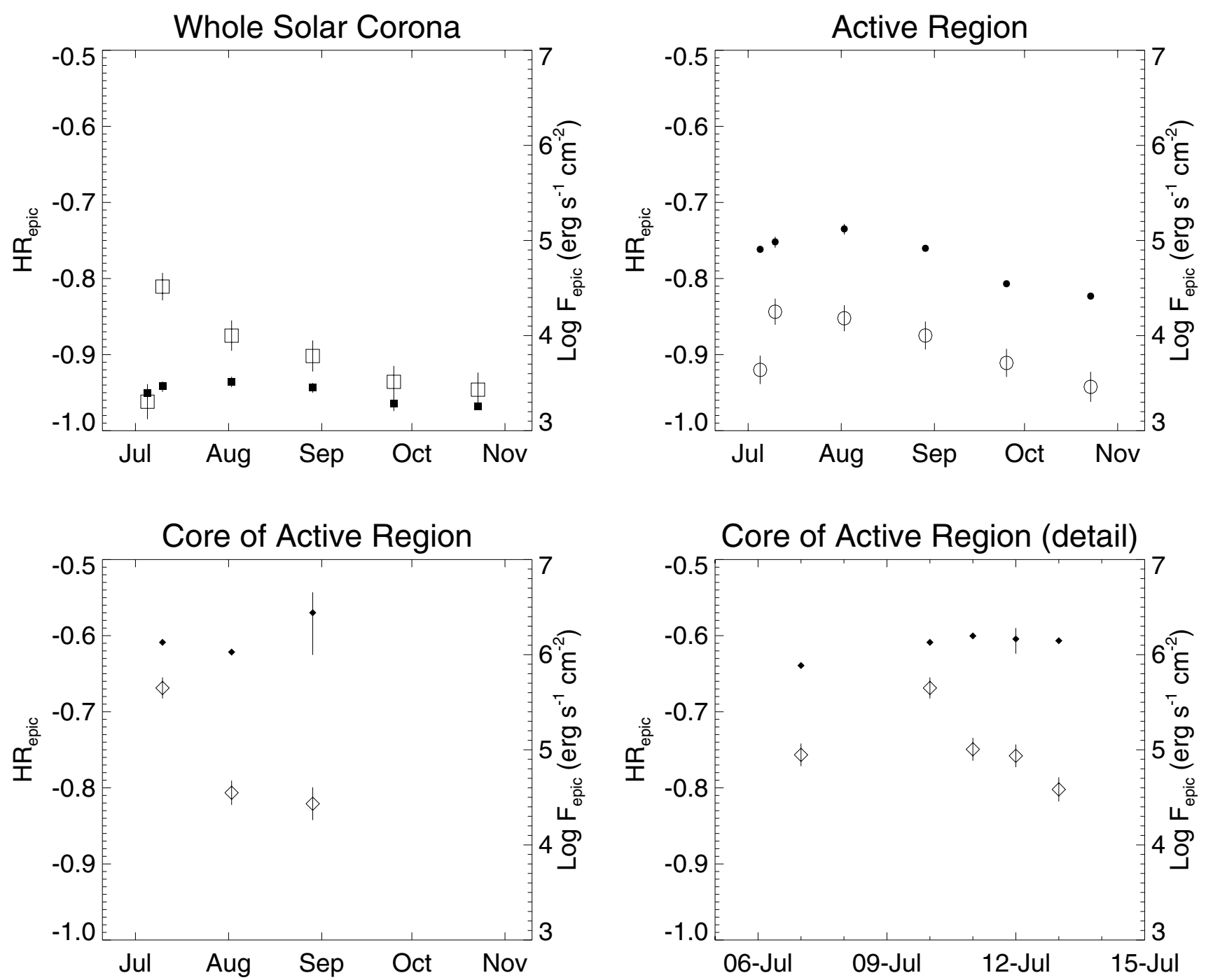

Fig. 5. Evolution of the hardness ratio (open symbols) and X-ray surface flux (filled symbols) - in the XMM-Newton/EPIC band - of the whole solar corona (squares), of the AR (circles), and of the core (diamonds) from the emergence to the decay of the active region. The lower right panel shows more in detail the evolution of the core during the emergence phase (July 7-14, 1996); data points on July 10, 1996 are reported in both the lower left and right panels.

its activity is largely due to the young active regions present on the disk.

\subsubsection{Rotational modulation}

While the background corona provides the main contribution to the whole corona $\operatorname{EM}(T)$ distribution around $T \sim 10^{6} \mathrm{~K}$, active regions and cores mainly contribute to higher temperatures (around $2-3 \times 10^{6} \mathrm{~K}$ ). We expect, therefore, that the periodic occultation of the only active region present - due to solar rotation - will modulate the high temperature portion of the $\operatorname{EM}(T)$ of the whole Sun. With only one active region, we are in the most favorable situation to detect rotational modulation. Figure 6 shows two examples of the $E M(T)$ distributions, derived from the SXT data at different phases of solar rotation, i.e. when AR 7978 was (July 11, 1996) and was not (September 10, 1996) visible on the solar disk. The two $E M(T)$ distributions have similar shapes but are quantitatively different: the latter $\operatorname{EM}(T)$ is lower at all temperatures with a steeper slope of the descending branch and has no plasma with $T \geq 3 \times 10^{6} \mathrm{~K}$. These variations are very similar to those observed during the solar cycle discussed in Peres et al. (2000). As a consequence of these changes, the corresponding stellarlike X-ray spectra should vary significantly.

As a first step, we derived the X-ray luminosity, $L_{\mathrm{pspc}}$, of the whole corona in the Rosat/PSPC spectral band. We found that the rotational modulation leads to variations of $L_{\mathrm{pspc}}$ between 1 and $7 \times 10^{26} \mathrm{erg} \mathrm{s}^{-1}$; such values are only slightly higher than that of the background corona (in the range $0.6-3 \times$ $10^{26} \mathrm{erg} \mathrm{s}^{-1}$; see Paper IV), indicating that the contribution of the active region to the whole corona $L_{\mathrm{pspc}}$ is small during its entire evolution. Figure 3 explains this result, showing that the $E M_{\text {sum }}$ of the AR and of the core are both generally lower than the $E M_{\text {sum }}$ of the whole corona.

Figure 7 shows the spectral hardness of the whole corona, $H R$, and the surface brightness in the instrument's 


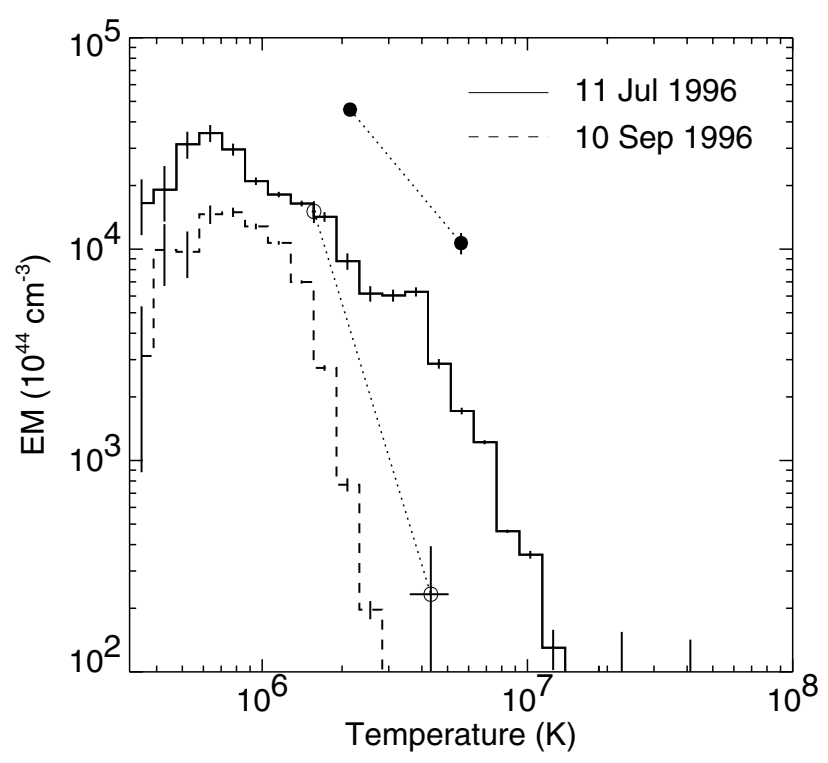

Fig. 6. Examples of $E M(T)$ distributions of the whole solar corona observed on July 11, 1996 (solid line), during the emergence of the active region, and on September 10, 1996 (dashed line), when no active region was present. The figure also shows the results of the spectral fitting to the Chandra/ACIS spectra (including error bars) for the two examples (filled and open circles respectively).

band, $F_{\mathrm{X}}$, derived for Rosat/PSPC, XMM-Newton/EPIC and Chandra/ACIS, over the whole time interval under analysis, sampled at intervals of four days (see Sect. 2.1). Both $H R$ and $F_{\mathrm{X}}$ of the whole corona change because of the rotational modulation for the three instruments considered: $F_{\mathrm{X}}$ varies by up to one order of magnitude in the Chandra/ACIS band, and $H R$ by up to a factor 2 (corresponding to variations of the average temperature by a factor 4). It appears that Chandra/ACIS is significantly more sensitive to coronal changes due to rotational modulation than the other two instruments. However, the higher sensitivity of $H R_{\text {acis }}$ to rotational modulation is just due to the harder band we have selected; selecting the same bands for XMM-Newton/EPIC we found analogous results as for Chandra/ACIS and the evolution of this alternative choice of $H R_{\text {epic }}$ is very similar to that of $H R_{\text {acis }}$ with just an overall shift of $\sim 0.2$ along the $H R$ axis. The rotational modulation due to a single active region on the disk can lead to significant variability of the observed X-ray flux with only moderate changes of the spectral hardness in the Rosat/PSPC and XMM-Newton/EPIC data and significant $F_{\text {acis }}$ and $H R_{\text {acis }}$ changes in the Chandra/ACIS observations.

This result can be understood in the light of the changes in the whole corona $\operatorname{EM}(T)$ distribution induced by the rotational modulation (see Fig. 6). In particular, the large variations of the total emission measure cause the significant variations of the X-ray flux. The relative weight of the hot plasma increases when the active region is visible on the disk, leading to a moderate increase of the emission-measure-weighted temperature and to a moderate variability of the hardness ratios.

The above discussion also explains why the X-ray flux in the Chandra/ACIS band is lower than that in the Rosat/PSPC and XMM-Newton/EPIC bands and why the variations of $F_{\text {acis }}$ due to the rotational modulation are more evident: the Chandra/ACIS band is slightly harder than those of the other two instruments, so it is less sensitive to low temperature plasma in the solar corona and more sensitive to the changes of $\operatorname{EM}(T)$ at high temperatures. Since the rotational modulation leads to large variations of emission measure at high temperatures, Chandra/ACIS spectra are expected to show high variability of $H R_{\text {acis }}$ and $F_{\text {acis }}$. As discussed before, a choice of bands for XMM-Newton/EPIC similar to those of Chandra/ACIS leads to similar results.

It is interesting to investigate the effect of the rotational modulation on the multi-thermal components used for fitting the stellar-like spectra. We discuss here, as an example, the results for Chandra/ACIS, which among the instruments considered is the most sensitive to the rotational modulation. Figure 8 shows the temperature of the cold, $T_{\text {cold }}$, and the hot, $T_{\text {hot }}$, isothermal components fitting the Chandra/ACIS spectra and the ratio of the hot to the cold EM components, $E M_{\text {hot }} / E M_{\text {cold }}$. The effect of the rotational modulation is evident in the ratio $E M_{\text {hot }} / E M_{\text {cold }}$ which varies by up to two orders of magnitude; the two temperatures vary in phase with $E M_{\text {hot }} / E M_{\text {cold }}$ but with a smaller amplitude, within a factor 1.7 for the cold component and a factor 2.0 for the hot component: the different weights of plasma at different temperatures determines the modulation. This can be explained comparing again the $E M(T)$ distributions derived from the SXT data at different rotation phases (see Fig. 6). The filled and open circles in the figure mark the emission measure and temperatures values of the isothermal components used for fitting the corresponding Chandra/ACIS spectrum. As discussed above, both distributions have a maximum around $10^{6} \mathrm{~K}$, but the slope of the descending branch of the distribution gets steeper when no active regions are visible, because their contribution (mainly at high temperatures) is missing and, therefore, the hot isothermal component has a low relative weight.

\section{Stellar-like $H R-F_{X}$ diagrams}

One of the main implications of the analysis presented so far is that we are now in a condition to compare the X-ray variability induced by rotational modulation with the variability caused by other phenomena occurring in the solar corona, in particular by the solar cycle and by the flares.

The X-ray variability of the non-flaring Sun induced by its 11-years cycle of activity has been studied in Paper IV with SXT data collected between December 1991 and April 1996, i.e. from the maximum to the minimum of solar cycle 22 . There, we discussed the X-ray variability in the Rosat/PSPC band and we found a correlation between $F_{\mathrm{pspc}}$ and $H R_{\mathrm{pspc}}$, with $F_{\text {pspc }}$ varying by up to 2 orders of magnitude.

The X-ray variability of the whole corona due to a solar flare was first analyzed in Paper II. In the following we include a more thorough analysis, considering two extreme cases, the Sun close to the minimum (July 10, 1996) and close to the maximum (January 6, 1992) of its cycle, and including the flare evolution. We have considered the sample of eight flares analyzed in Paper III, ranging from weak (GOES class C5.8) to very intense ones (X9), and we investigated the variability 

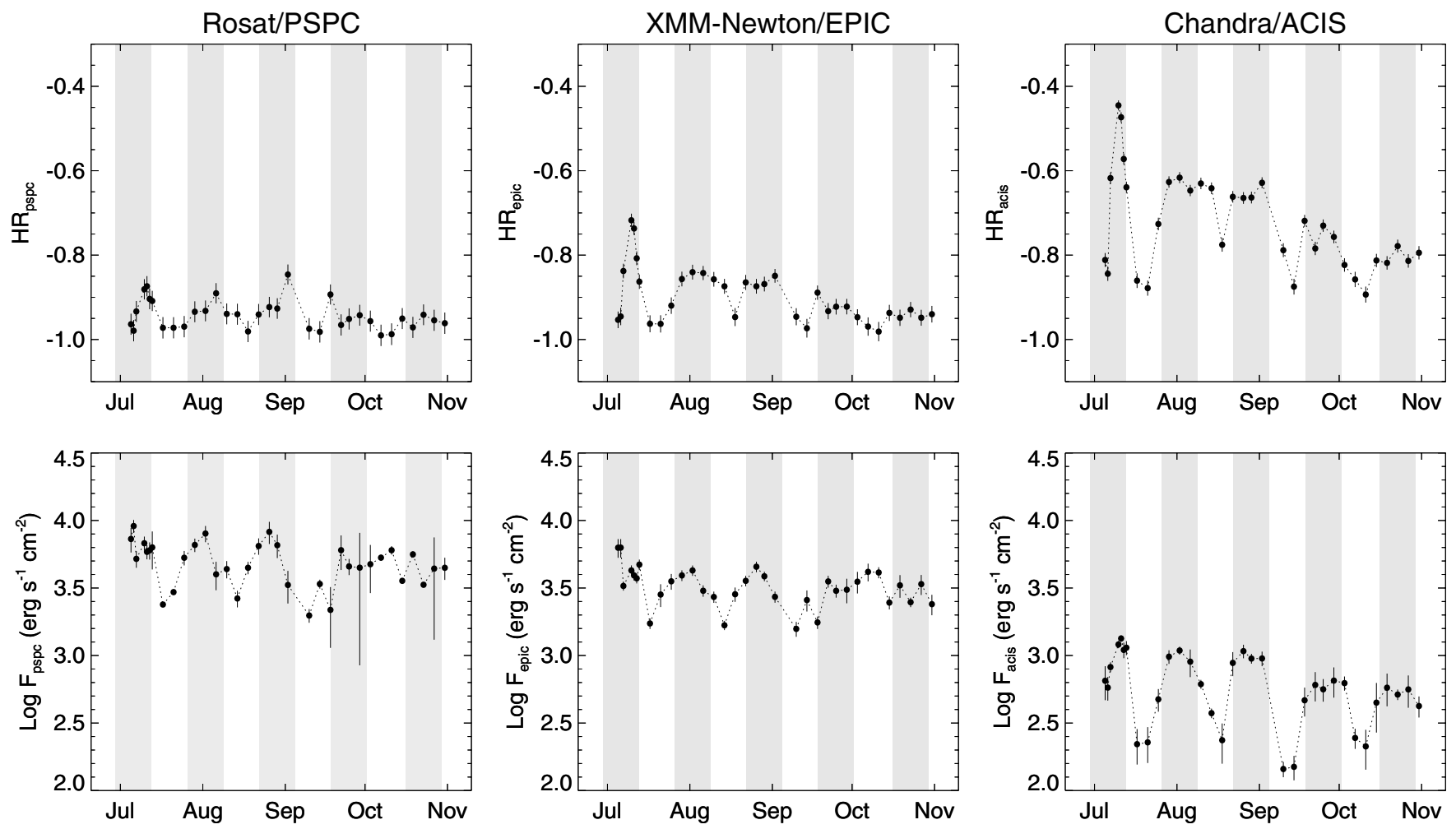

Fig. 7. Hardness ratio (upper panels) and X-ray surface flux (lower panels) in the Rosat/PSPC, XMM-Newton/EPIC, and Chandra/ACIS bands of the whole solar corona vs. time (one data point every four days with the only exception of July 1996 which was sampled more frequently to show the emergence of the active region). The gray stripes mark the times when AR 7978 was visible on the disk.

induced by them in the X-ray emission of the whole solar corona. In Paper III, however, we isolated the flares from the corona, to study more in detail the relevant plasma effects. Here we added the flares to the corona (as in Paper II), considering the corona both at maximum and at minimum of the cycle. In other words, the method used here accounts for the flare and the quiescent corona, as in Paper II, and considers the flare evolution, as in Paper III. In our approach we first sample the $E M(T)$ profiles of the Sun plus flare at constant intervals of $60 \mathrm{~s}$; we then synthesize the spectrum from each of them and fold it through the spectral response of Rosat/PSPC, XMM-Newton/EPIC, and Chandra/ACIS; after binning each sequence of observations into three or longer time segments (lasting between $180 \mathrm{~s}$ and $3000 \mathrm{~s}$ ), we sum all folded spectra, within each time bin, into a single spectrum; each of these spectra is normalized to yield a total number of counts typical of a good stellar observation (e.g. between 3000 and 5000 counts for Rosat/PSPC, and between 1000 and 10000 counts for XMM-Newton/EPIC and Chandra/ACIS).

\subsection{Rosat/PSPC}

Combining all the data from the study of the active region evolution and of the rotational modulation, those on the two corona-plus-flare realizations, with those on the variability due to the activity cycle (Paper IV) in a $F_{\mathrm{pspc}}$ vs. $H R_{\mathrm{pspc}}$ diagram, we can compare the features of the three kinds of variability (see Fig. 9). As discussed in Sect. 3.2.2, the rotational modulation due to one active region leads to significant changes in the flux $F_{\mathrm{pspc}}$ (up to a factor 6 in the Rosat/PSPC band), while the hardness ratio changes only slightly in the PSPC band.

The solar cycle is characterized by X-ray variability with small changes of $H R_{\mathrm{pspc}}$ but large changes of $F_{\mathrm{pspc}}$ : the filled circles in Fig. 9 mark the position of the non-flaring Sun from the maximum to the minimum. The X-ray variability across the four rotations analyzed in this paper is characterized by a correlation between $F_{\mathrm{pspc}}$ and $H R_{\mathrm{pspc}}$ similar to that across the cycle. In both cases, the peak temperature of the $E M(T)$ ranges between $\sim 8 \times 10^{5} \mathrm{~K}$ and $\sim 2 \times 10^{6} \mathrm{~K}$ and, in the EM, the weight of hot plasma $\left(>3 \times 10^{6} \mathrm{~K}\right)$ relative to that of the entire corona changes significantly.

Our study allows us to derive an upper limit to the rotational modulation we could observe with Rosat/PSPC on a star similar to the Sun, assuming that one side is covered with active regions as during the solar cycle maximum and the other is without active regions. In the Rosat/PSPC case, for instance, Fig. 9 clearly shows that, even in this hypothetical case, the X-ray flux, $F_{\mathrm{pspc}}$, of the non-flaring Sun could change by two orders of magnitude, from $10^{3}$ to $10^{5} \mathrm{erg} \mathrm{s}^{-1} \mathrm{~cm}^{-2}$, whereas the hardness ratio, $H R_{\mathrm{pspc}}$, would range only between -1.0 and -0.7 . Even in this extreme case, therefore, our analysis shows that the X-ray rotational modulation can be detected with Rosat/PSPC through significant X-ray flux variability without large spectral variations.

Figure 9 also shows the variability due to flares. Remarkably enough, all the simulations pertaining to the flares plus corona at maximum (open circles) align along a well defined track, and the analogous simulations with the Sun at 


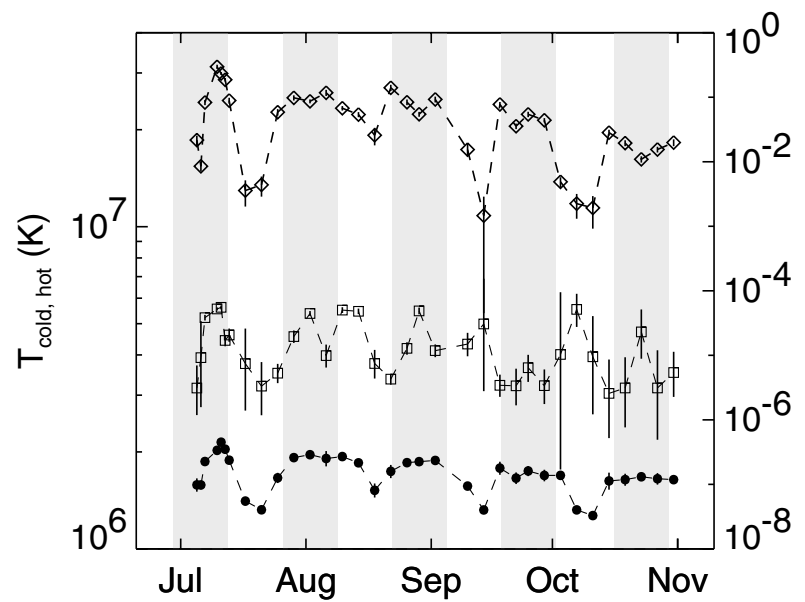

Fig. 8. The temperature of the cold (filled circles) and hot (squares) thermal components fitting the Chandra/ACIS spectra and of the ratio (diamonds) of the emission measure of the hot to the cold fitting component vs. time.

minimum (crossed circles) along another almost paralleltrack, at lower flux. Both at the maximum and minimum of the solar cycle, a flare can change the $H R_{\mathrm{pspc}}$ significantly, making the Rosat/PSPC spectrum much harder than that of the non-flaring Sun: close to the maximum, $H R_{\mathrm{pspc}}$ can increase from -0.8 to -0.1 and $F_{\text {pspc }}$ by a factor $\sim 4$; close to the minimum, $H R_{\mathrm{pspc}}$ can increase from -0.9 to 0.2 and $F_{\mathrm{pspc}}$ by 2 orders of magnitude. Therefore, at variance with the variability due to the modulation or to the cycle, the flare variability involves strong variations of both $F_{\mathrm{pspc}}$ and $H R_{\mathrm{pspc}}$. This can be explained in the light of the results of Paper III, which showed that a flare leads to a double-peaked $E M(T)$ distribution with one peak (due to the non-flaring corona) at a few $10^{6} \mathrm{~K}$, and the other peak (due to the flare) around $10^{7} \mathrm{~K}$ (see also Paper II). Near the flare maximum, the relative contribution of the hot plasma around $10^{7} \mathrm{~K}$ can be larger, leading to higher values of both $F_{\mathrm{pspc}}$ and $H R_{\mathrm{pspc}}$.

Our main conclusion is that the rotational modulation and the solar cycle lead to X-ray variability in the Rosat/PSPC band characterized by the same slope in the $H R_{\mathrm{pspc}}-F_{\mathrm{pspc}}$ diagram; the rotational modulation, in particular, causes a small variation of $H R_{\mathrm{pspc}}$. The $\mathrm{X}$-ray variability induced by flares instead has a flatter slope in the $H R_{\mathrm{pspc}}-F_{\mathrm{pspc}}$ diagram and significant changes in $H R_{\mathrm{pspc}}$.

\subsection{XMM-Newton/EPIC and Chandra/ACIS}

We derived $H R-F_{\mathrm{X}}$ diagrams also for the XMM-Newton/EPIC and Chandra/ACIS spectra (see Eq. (2) for the definition of $H R$ in these cases), and, again, we found that Chandra/ACIS is more sensitive to coronal changes than the other two instruments, showing larger variations of $H R$.

As already discussed in Sect. 3.2, XMM-Newton/EPIC and Chandra/ACIS have a good spectral resolution, adequate for investigating the changes in the relative weight of the fitting isothermal components. Figure 10 shows the ratio of the emission measure of the hot component fitting the

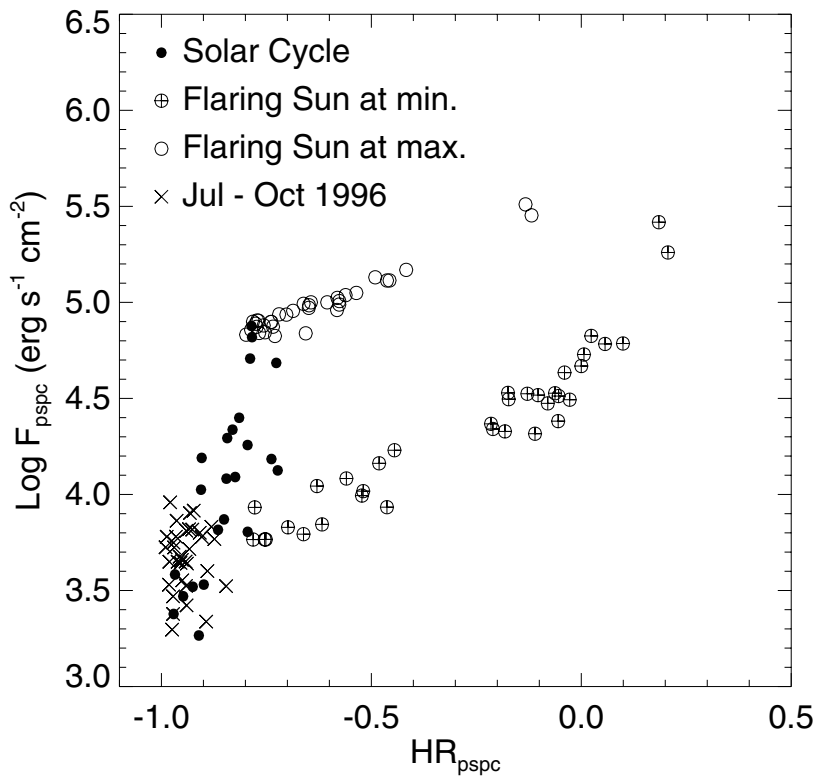

Fig. 9. X-ray surface flux (erg s $\mathrm{s}^{-1} \mathrm{~cm}^{-2}$ ) in the Rosat/PSPC band vs. the Rosat/PSPC hardness ratio. The figure shows the result for the non-flaring solar corona during the four rotations analyzed (crosses), during the solar cycle (filled circles; from Paper IV) and for the corona with flares at activity minimum (crossed circles) and maximum (open circles).

Chandra/ACIS spectra over that of the cold component, $E M_{\text {hot }} / E M_{\text {cold }}$, vs. $H R_{\text {acis. }}$. All the cases show correlation between $E M_{\text {hot }} / E M_{\text {cold }}$ and $H R_{\text {acis }}$ : the higher the hardness ratio, $H R_{\text {acis }}$, the higher the relative weight of the hot component.

Analogously to the Rosat/PSPC case, the X-ray variability induced by the solar cycle and by the rotational modulation in the XMM-Newton/EPIC spectra leads to small variations of both $H R_{\text {epic }}$ (ranging between -0.1 and -0.7 ) and $E M_{\text {hot }} / E M_{\text {cold }}$ (between 0.02 and 0.4 ); the X-ray variability induced during the evolution of a flare leads to large variations of both $H R_{\text {epic }}$ (ranging between -0.8 and 0.2 ) and $E M_{\text {hot }} / E M_{\text {cold }}$ (between 0.2 and 30 ) with a flatter correlation.

A similar result has been found with Chandra/ACIS; the rotational modulation and the solar cycles lead to X-ray variability with similar characteristics: moderate changes of $H R_{\text {acis }}$ (ranging between -0.9 and -0.4 ) and large changes of $E M_{\text {hot }} / E M_{\text {cold }}$ (between 0.003 and 0.5 ). The variability due to flares is instead characterized by larger variations of $H R_{\text {acis }}$ (ranging between -0.5 and 0.7 ; see Fig. 10), as we found in Sect. 4.1. Typically, the changes in $H R_{\text {acis }}$ are significantly larger than those for the other two detectors, albeit this is due to the harder band chosen; choosing the same bands for XMM-Newton/EPIC gave analogous results.

Marino et al. (2003) analyzed XMM-Newton/EPIC observations of VXR45, a young fast-rotating star member of IC 2391 and found evidence of X-ray rotational modulation characterized by small spectral variability, analogously to what we found here (see also Sect. 3.2.2). In the light of our findings, their result can be explained by a variation of the fraction of the stellar surface covered with active regions. Our analysis, 


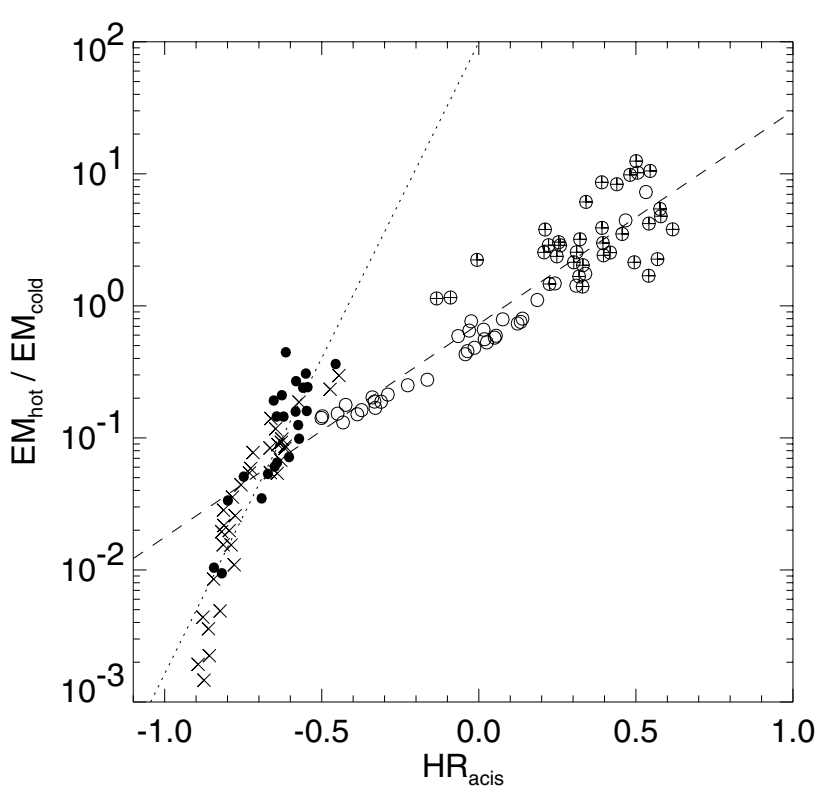

Fig. 10. The ratio of the emission measure of the hot component fitting the Chandra/ACIS spectra and that of the cold component vs. the Chandra/ACIS hardness ratio. The symbols are as in Fig. 9. The leastsquares fit was made to data on the solar cycle variations and on the four solar rotations analyzed (dotted line) and data on flares (dashed line).

therefore, is in agreement with the $F_{\mathrm{X}}$ and spectral findings by Marino et al. (2003).

\section{Summary}

This paper is part of a project aimed at studying the Sun as an X-ray star and at comparing solar and stellar observations. In the present work, we focused on the evolution of an active region observed during the minimum of the solar cycle, aiming at:

1. studying how the active region contributions to the whole corona X-ray $\operatorname{EM}(T)$, spectrum and luminosity evolve;

2. studying the X-ray variability induced by the rotational modulation on a star like the Sun.

To this end, we have analyzed a sample of SXT data collected between July and October 1996, monitoring most of the evolution of the active region AR 7978 from its emergence to its decay. The evolution of the active region has been analyzed using the SXT observations during its emergence and its successive CMPs; the rotational modulation has been analyzed using the SXT observations collected with a cadence of four days. Whenever the active region was visible on the disk, we have selected the core and the intermediate brightness portion of the active region, with a criterion based on the SXT pixel intensity. We have studied the evolution of the $E M(T)$ distributions of these two classes of X-ray solar structures and for the whole solar corona, and of the corresponding focal-plane spectra that non-solar X-ray observatories (Rosat, XMM-Newton, and Chandra) would collect observing a star like the Sun.

Our analysis has shown that the $E M(T)$ distribution of an active region is subject to significant variations during its evolution, in particular: i) the related average temperature, $\langle T\rangle$, has a maximum few days after its emergence and then it slowly decreases with decay of the active region; ii) the total emission measure, $E M_{\text {sum }}$, first increases, reaching a maximum approximately one month after the emergence of the active region (interestingly enough after maximum $\langle T\rangle$ ) and then decreases by more than one order of magnitude in two months as an effect of the decay of the active region. The core evolves more rapidly and contributes to an increase of the whole corona $E M(T)$ distribution only at the emergence of the active region; in few days $\langle T\rangle$ and $E M_{\text {sum }}$ of the core reach a maximum and then decrease till the core disappears, roughly one month later. The $E M_{\text {sum }}$ of the core is approximately as high as that of the rest of the active region during the emergence, so that their contribution to the whole corona $E M(T)$ is of equal importance. Furthermore, we found that they contribute to the $E M(T)$ distribution, X-ray luminosity and spectrum of the whole solar corona especially when the active region is young.

We also analyzed the X-ray rotational modulation induced when only one active region is present on the Sun. We found this simple case particularly useful because of its general implications for stellar X-ray rotational modulation, and we believe that it can be used as a guide and a template to interpret more complex cases. The rotational modulation is characterized by significant variations of the $E M(T)$ distribution of the whole solar corona; the average temperature $\langle T\rangle$ instead does not change much. As derived from the corresponding stellar-like spectra, the variations of the X-ray flux are significant (up to one order of magnitude in the Chandra/ACIS band) and the variations of the hardness ratio are small in the Rosat/PSPC and XMM-Newton/EPIC bands but significantly larger in the Chandra/ACIS bands (or equivalent bands selected for XMM-Newton/EPIC).

Finally, we compared the characteristics of X-ray variability due to the rotational modulation, to the solar cycle and to the flares in the solar corona in order to have some hints for the interpretation of the stellar X-ray variability. In the Rosat/PSPC case, we found that the variability induced by rotational modulation and that by the solar cycle are both characterized by a similar correlation between the X-ray flux, $F_{\mathrm{pspc}}$, and the relevant hardness ratio, $H R_{\mathrm{pspc}}$, (albeit on very different time scales) with a steep slope due to the small variation of $H R_{\mathrm{pspc}}$. On the other hand, the variability due to the evolution of a flare has completely different characteristics in the $F_{\mathrm{pspc}^{-}}$ $H R_{\mathrm{pspc}}$ diagram: the correlation between $F_{\mathrm{pspc}}$ and $H R_{\mathrm{pspc}}$ has a flatter slope and, in general, the hardness ratio reaches high values when the flare is close to its maximum intensity. In the case of XMM-Newton/EPIC and Chandra/ACIS, we derived the diagram $E M_{\text {hot }} / E M_{\text {cold }}$ vs. $H R$ and we found a correlation between $E M_{\text {hot }} / E M_{\text {cold }}$ and $H R$ with one slope for the rotational modulation and the solar cycle and with a flatter slope for the flares.

This work provides a template to interpret the X-ray variability detected in stellar data in terms of phenomena observed on the Sun, and allows us to relate the characteristics observed in stellar X-ray spectra to the structures studied in the whole corona, their $\operatorname{EM}(T)$, and their plasma characteristics. 
Acknowledgements. We thank the anonymous referee for his/her comments on the manuscript. This work was supported in part by Agenzia Spaziale Italiana and by Ministero dell'Istruzione, dell'Università e della Ricerca.

\section{References}

Arnaud, K. 1996, Astronomical data analysis software and systems, ed. G. Jacoby, \& J. Barnes, ASP Conf. Ser., 101, 14

Culhane, J. L., Bently, R. D., Hiei, E., et al. 1991, Sol. Phys., 136, 89

Dèmoulin, P., van Driel-Gesztelyi, L., Mandrini, C. H., Klimchuk, J. A., \& Harra, L. 2003, ApJ, 586, 592

Drake, J. J., Peres, G., Orlando, S., Laming, J. M., \& Maggio, A. 2000, ApJ, 545, 1074

Fludra, A., Bentley, R. D., Lemen, J. R., Jakimiec, J., \& Sylwester, J. 1989, ApJ, 344, 991

Golub, L., Noci, G., Poletto, G., \& Vaiana, G. S. 1982, ApJ, 259, 359

Harvey, K. L., \& Hudson, H. S. 1998, in Observational plasma astrophysics: five years of Yohkoh and beyond, ed. T. Watanabe, T. Kosugi, \& A. C. Sterling (Kluwer Academic Publischers), 315

Hudson, H. S., Labonte, B. J., Sterling, A. C., \& Watanabe, T. 1998, in Observational plasma astrophysics: five years of Yohkoh and beyond, ed. T. Watanabe, T. Kosugi, \& A. C. Sterling (Kluwer Academic Publischers), 237

Kaastra, J. S. 1992, An X-ray spectral code for optically thin plasmas, Internal SRON-Leiden Report, updated version 2.0

Lang, J., Bentley, R. D., Brown, C. M., et al. 1992, PASJ, 44, L55

Marino, A., Micela, G., Peres, G., \& Sciortino, S. 2003, A\&A, 407, L63

McCann, S. M., \& Keenan, F. P. 1988, J. Phys. Colloq. C1 France, 49, $\mathrm{C} 1$
Mewe, R., Gronenschild, E. H. B. M., \& van den Oord, G. H. J. 1985 , A\&AS, 62, 197

Micela, G., Pye, J., \& Sciortino, S. 1997, A\&A, 320, 865

Morrison, M. 1994, Yohkoh analysis guide. vol. 1: User's Guide

Ogawara, Y., Takano, T., Kato, T., et al. 1991, Sol. Phys., 136, 1

Oláh, K., van Driel-Gesztelyi, L., Kövári, Z., \& Bartus, J. 1999, A\&A, 344, 163

Orlando, S., Peres, G., \& Reale, F. 2000a, ApJ, 528, 524

Orlando, S., Khan, J., van Driel-Gesztelyi, L., et al. 2000b, Adv. Space Res., 25, 1913

Orlando, S., van Driel-Gesztelyi, L., Thompson, B., Khan, J., \& Foing, B. H. 2000c, in Variable stars as essential astrophysical tools, NATO Science Ser.: C, Mathematical and Physical Sciences, ed. C. Ibanoglu, 544, 783

Orlando, S., Peres, G., \& Reale, F. 2001, ApJ, 560, 499

Peres, G., Orlando, S., Reale, F., Rosner, R., \& Hudson, H. 2000, ApJ, 528,537

Peres, G., Orlando, S., \& Reale, F. 2004, ApJ, in press

Reale, F., Peres, G., \& Orlando, S. 2001, ApJ, 557, 906

Schmitt, J. H. M. M. 1997, A\&A, 318, 215

Sterling, A. C., Hudson, H. S., \& Watanabe, T. 1997, ApJ, 479, L149

Svestka, Z. 1976, in Solar Flares (Dordrecht: Reidel)

Tsuneta, S., Acton, L., Bruner, M., et al. 1991, Sol. Phys., 136, 37

van Driel-Gesztelyi, L. 1998, Proc. 2nd ASPE, ed. C. Alissandrakis, \& B. Schmieder, ASP Conf. Ser., 155, 202

van Driel-Gesztelyi, L., Mandrini, C., Thompson, B., et al. 1999, Proc. 3rd ASPE, ed. B. Schmieder, A. Hofmann, \& J. Staude, ASP Conf. Ser., 184, 302

van Driel-Gesztelyi, L., Dèmoulin, P., Mandrini, C. H., Harra, L., \& Klimchuk, J. A. 2003, ApJ, 586, 579 